\title{
The Effects of a PPAR $\alpha$ Agonist on Myocardial Damage in Obese Diabetic Mice With Heart Failure
}

\author{
Rui Chen, ${ }^{1,5^{*}} \mathrm{MD}$, Fengxia Liang, ${ }^{2,7^{*}} \mathrm{MD}$, Shigeto Morimoto, ${ }^{3} \mathrm{MD}$, Qian Li, ${ }^{6} \mathrm{MD}$, \\ Junji MoriYa, ${ }^{1}$ MD, Jun-ichi Yamakawa, ${ }^{1}$ MD, Takashi TaKahashi, ${ }^{1}$ MD, Kunimitsu IwaI, ${ }^{3}$ MD, \\ and Tsugiyasu KANDA, ${ }^{4} \mathrm{MD}$
}

\begin{abstract}
SUMMARY
Recent studies have confirmed that PPAR $\alpha$ agonists have not brought the anticipated benefits to patients with type 2 diabetes and potentially fatal heart disease. We hypothesized that such agonists may have a cardio-suppressive effect in treating such disorders, therefore, we inoculated diabetic KKAy mice with encephalomyocarditis virus (EMCv) to induce a diabetic model with severe myocardial damage. WY14643, a potent PPAR $\alpha$ agonist, was administered intraperitoneally either simultaneously (WY14643-late group) or 3 days before viral inoculation (WY14643-early group). WY14643-treated mice, especially those in the WY14643-early group, had higher mortality than those in the vehicle-treated group (vehicle) in the first 5 days after EMCv inoculation. However, the survival rate in the vehicle group decreased rapidly after day 4 and was the lowest of all 3 groups by day 9 . The WY14643-treated mice showed reduced body weight and blood glucose, improved myocardial pathological changes, lower cardiac TNF- $\alpha$ expression, and significantly higher adiponectin expression, whereas the LW/LC ratio was lower and cardiac UCP3 mRNA expression higher in the WY14643 treatment groups than in the vehicle group on day 4. WY14643 therefore has cardioprotective and cardio-suppressive effects when used to treat EMCvinduced myocarditis in diabetic mice. The cardioprotective effect may be due to its anti-inflammatory properties and its ability to increase cardiac adiponectin expression, whereas the reduced cardiac efficiency may be due to its enhancement of cardiac UCP3 mRNA expression. (Int Heart J 2010; 51: 199-206)
\end{abstract}

Key words: Tumor necrosis factor- $\alpha$ (TNF- $\alpha$ ), Adiponectin, Uncoupling protein 3 (UCP3)

$\mathrm{P}$ roxisome proliferator-activated receptor $\alpha(\operatorname{PPAR} \alpha)$ belongs to the nuclear receptor superfamily and is known to regulate the expression of genes for the transport proteins and enzymes that participate in inflammation and metabolism. ${ }^{1)}$ It has been shown that PPAR $\alpha$ agonists are capable of decreasing the production of some inflammatory cytokines, such as tumor necrosis factor- $\alpha$ (TNF- $\alpha$ ) in cardiac myocytes. ${ }^{2)}$ Moreover, the potent PPAR $\alpha$ agonist WY14643 can directly increase the expression of adiponectin receptors in white adipose tissue, which bind with adiponectin and exert antidiabetic, antiatherosclerotic, and anti-inflammatory effects. ${ }^{3)}$ Likewise, recent reports have indicated that PPAR $\alpha$ agonists can improve the survival rate of experimental animals with heart failure.4)

The Fenofibrate Intervention and Event Lowering in Diabetes (FIELD) study, however, found that PPAR $\alpha$ agonists had not brought the anticipated benefits to heart failure in type 2 diabetic patients and had even increased the death rate due to fatal cardiac disease, although to a statistically insignificant degree, ${ }^{5)}$ thus suggesting that $\operatorname{PPAR} \alpha$ agonists may have some adverse effects on myocardial damage or cardiac function despite their cardioprotective effects.

Uncoupling proteins (UCPs) are inner mitochondrial membrane proteins that play a role in dissipating the mitochondrial proton gradient by allowing protons to re-enter the mitochondrial matrix without the concomitant synthesis of ATP. Three such proteins-UCP1, UCP2, and UCP3are known. ${ }^{\text {) }}$ We are interested in UCP3 because it is mainly expressed in heart and skeletal muscle and is involved in the regulation of biological processes associated with mitochondrial energy metabolism. Indeed, increased UCP3 levels have been reported to correlate with higher myocardial consumption and reduced cardiac efficiency. ${ }^{8)}$ WY 14643 can increase the level of UCP3 mRNA in liver and brown fat tissue of KKAy mice. ${ }^{3)}$ Likewise, myocardial levels of UCP3 in BALB/c mice increased by $54 \%$ upon treatment with WY14643. ${ }^{9)}$ WY14643 may therefore exacerbate heart failure by increasing UCP3 expression, although more evi-

From the ${ }^{1}$ Departments of General Medicine, ${ }^{2}$ Endocrinology, ${ }^{3}$ Geriatric Medicine and ${ }^{4}$ Community Medicine, Himi City Hospital, Kanazawa Medical University, Toyama, Japan ${ }^{5}$ Department of Traditional Chinese Medicine, Union Hospital, ${ }^{6}$ School of Public Health, Tongji Medical College, Huazhong University of Science and Technology, and ${ }^{7}$ Hubei College of Traditional Chinese Medicine, Wuhan, China. ${ }^{*}$ These authors contributed equally to this work.

This study was supported in part by a grant for Promoted Research from Kanazawa Medical University (S2005-5), a Grant-in-Aid for Scientific Research (C) from the Ministry of Education, Science, Sports, and Culture of Japan (no. 17590767), and a grant from the Chinese Postdoctoral Science Foundation (no. 20070420179). This work was supported by a Grant-in-Aid for Scientific Research from the Ministry of Education, Science, Sports and Culture of Japan to T.K. (no. 19590835) and by a Grant for Project Research of Kanazawa Medical University (H2010-13).

Address for correspondence: Tsugiyasu Kanda, MD, Department of Community Medicine, Himi City Hospital, Kanazawa Medical University, 31-9 Saiwaicho, Himi, Toyama 935-8531, Japan.

Received for publication September 10, 2009.

Revised and accepted February 19, 2010. 
dence is needed in the event of diabetes complicated with fatal heart disease.

Some metabolic diseases, such as type 2 diabetes and obesity, predispose to heart failure, and inflammation plays an important role in the association between them. ${ }^{10)}$ Encephalomyocarditis virus (EMCv) can induce severe myocarditis and heart failure in experimental animals. ${ }^{11)}$ In previous studies, we inoculated EMCv into obese mice to set up a model of obese mice with severe heart failure, and found higher TNF- $\alpha$ and lower adiponectin expression levels in the injured myocardium. ${ }^{12,13)}$ Herein, obese diabetic KKAy mice inoculated with EMCv were used to induce a model of type 2 diabetes and obesity complicated with severe heart failure, and subsequently to evaluate the effect of WY14643. We determined the survival rate and examined the expression levels of cardiac TNF- $\alpha$, adiponectin, and UCP3 at different stages of myocardial damage and found that WY14643 plays different roles in the injured heart.

\section{MethodS}

Animals and treatments: Eight-week-old female KKAy mice weighing 38-42 g were purchased from Clea Japan Inc. (Tokyo), and maintained with food and water ad libitum. These mice were randomly divided into 3 groups: a WY14643-early group, which received WY14643 (Sigma, USA) at a daily dose of $50 \mathrm{mg} / \mathrm{kg}$ starting 3 days before viral inoculation, and a WY14643-late group and vehicle treatment group (vehicle), which received WY14643 and vehicle (dimethyl sulfoxide), respectively, simultaneously with viral inoculation. WY14643 $(0.1 \mathrm{~mL})$ was administered intraperitoneally once daily. Experimental protocols were approved by the Animal Experimental Committee of Kanazawa Medical University.

Study design: The study consisted of two experiments. Experiment 1 was performed to determine the survival rate and any change in body weight (BW). In this experiment, 21,28 , and 19 mice were raised in the vehicle, WY14643-

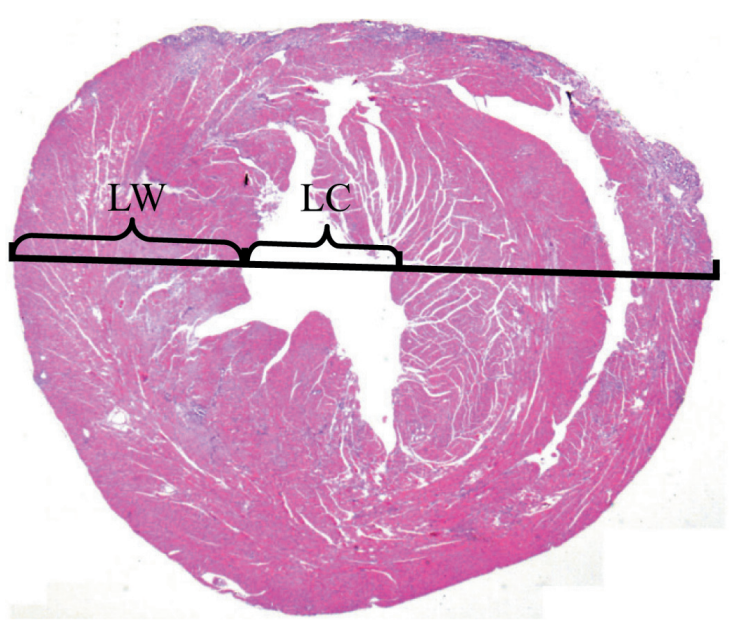

Figure 1. Measurement of left ventricular wall thickness and cavity dimension. LW indicates left ventricular wall and LC, left cavity. early, and WY14643-late groups, respectively. The survival rate and $\mathrm{BW}$ were recorded daily from 3 days before $\mathrm{EMCV}$ inoculation. The endpoint was a survival rate of less than $20 \%$ in any group. Experiment 2 was designed to obtain plasma and tissue samples on days 0,4 , and 9 after EMCv inoculation, with 45 mice in the vehicle group, 39 in the WY14643-early group, and 18 in the WY14643-late group. Eight KKAy mice without inoculation and treatment were used as normal control (control) and sacrificed on day 0 .

EMCv and inoculation protocol: A myocarditic variant of EMCv was provided by Dr. Y. Seto, Institute for Advanced Medical Research of Keio University (Tokyo). Virus preparations and inoculation were performed as described previously. $^{13)}$

Histological heart examinations: The BW and heart weight (HW) of each mouse was recorded at sacrifice in experiment 2 . The heart was transversely divided into two halves. One half, which included the cardiac apex, was stored at $-80^{\circ} \mathrm{C}$ until use, while the other half was fixed in $10 \%$ buffered formalin and blocked with paraffin for histological examination and immunohistochemical study. After fixation, the heart was transversely sectioned at the maximal circumference of the ventricle. Some slices were stained with hematoxylin-eosin to assess the severity of myocardial necrosis and inflammatory cell infiltration according to previously described scales. ${ }^{13,14)}$

The LV wall thickness (LW) and LV cavity (LC) dimensions were measured with the slice at the maximal circumference of the ventricle, as described by Matsumori, et $a l^{15)}$ (Figure 1). The diameter of the myocardial fiber in the lateral LV wall was determined as described previously. ${ }^{13)}$

Blood glucose (BG) and plasma free fatty acid (FFA): BG and plasma FFA were determined with a Fuji Dry Chem System (Medical System Co., Tokyo) and nonesterified fatty acid-C test kit (Wako Pure Chemical Industries, Osaka, Japan), respectively.

Immunohistochemical examination: To identify the anatomic localization and expression levels of TNF- $\alpha$, adiponectin, and UCP3 within the myocardium, immunohistochemistry was performed using the avidin biotin complex methods (Vectastain ABC kit, Vector Laboratories, Burlingame, CA), as described previously. ${ }^{14)}$ All sections were previously blocked with normal goat serum for 20 minutes at room temperature to minimize background staining. The slides were then incubated with anti-human TNF- $\alpha$ mouse monoclonal antibody clone 4H31 (\#HM2010, HyCult Biotechnology b.v., Uden, the Netherlands), anti-human adiponectin mouse monoclonal antibody clone (\#ab22554, Abcam, Tokyo), and UCP3 rabbit polyclonal antibody \#ab10985, Abcam, Japan). Sections were counterstained with hematoxylin. The slides were reviewed blind by the same pathologist and graded semiquantitatively according to the degree of immunoreactivity: 0 for absence of staining, 1 for weak, 2 for moderate, and 3 for strong staining. ${ }^{16}$ They were then compared with the respective control slides to exclude nonspecific staining.

Comparative expression of TNF- $\alpha$, adiponectin, and UCP3 mRNA in heart tissue: RNA extraction from the frozen cardiac tissue was performed following the manufacturer's protocol (RNeasy Mini Kit, QIAGEN Inc., Tokyo). DNAase was applied during RNA extraction to avoid DNA 
contamination. Total RNA concentration was determined by measuring the optical density at 260 and $280 \mathrm{~nm}$. Aliquots of $20 \mu \mathrm{L}$ RNA from each tissue sample were used to produce cDNA. Comparative expression levels of TNF- $\alpha$, adiponectin, and UCP3 mRNA in cardiac tissue from each group were determined using quantitative real-time reverse transcriptase-polymerase chain reaction (RT-PCR). A TaqMan minor groove binding (MGB) Probe (Applied Biosystems Inc., CA, USA) was applied for real-time PCR, and a commercially available kit was used for $\mathrm{TNF} \alpha$, adiponectin, and UCP3 RT-PCR (Applied Biosystems Inc.). Primers and TaqMan probes for the target gene (TNF $\alpha$, adiponectin, and UCP3) and internal reference gene (Rodent GAPDH) were purchased from Applied Biosystems (TaqMan ${ }^{\circledR}$ Gene Expression Assays). Each TaqMan probe was labeled with a reporter dye [6-carboxyfluorescein (FAM)] situated at the 5' end of the oligonucleotide and a quencher dye (MGB) located at the 3' end. TaqMan ${ }^{\circledR}$ Gene Expression Assay numbers for TNF, adiponectin, and UCP3 were Mm00443258mL, Mm00456425mL, and Mm00494074mL, respectively (Applied Biosystems Inc.). Quantification of target cDNA (TNF $\alpha$, adiponectin, and UCP3) and GAPDH was performed in 96-well plates using an ABI PRISM7500 Sequence Detection System (ABI); data collection and analysis was performed using the machine's software. PCR was carried out on a final volume of $25 \mu \mathrm{L}$ containing cDNA equivalent to $10-100 \mathrm{ng}$ of total RNA, $10 \mu \mathrm{L}$ of $2 \times$ TaqMan Fast PCR Master Mix, and $1 \mu \mathrm{L}$ of $20 \times$ TaqMan Expression Assay reagent. Each sample was analyzed in triplicate. The thermal cycler conditions were $95^{\circ} \mathrm{C}$ for 20 seconds, followed by 40 cycles at $95^{\circ} \mathrm{C}$ for 3 seconds, and $60^{\circ} \mathrm{C}$ for 30 seconds. The comparative $C_{T}$ method of data analysis was used to analyze the data. $\mathrm{C}_{\mathrm{T}}$ is the PCR cycle at which an increase in reporter fluorescence above the baseline level was first detected. $\mathrm{C}_{\mathrm{T}}$ values for the target and internal reference gene were calculated for each sample along with the difference between these values $\left(\Delta \mathrm{C}_{\mathrm{T}}\right) . \Delta \Delta \mathrm{C}_{\mathrm{T}}$ was calculated as the difference in $\Delta \mathrm{C}_{\mathrm{T}}$ between sample and calibrator sample. The amount of target gene expression, normalized to an internal reference and relative to calibrator, was given by: $2^{-\Delta \Delta \mathrm{CT}}$.

Statistical analysis: The Kaplan-Meier analysis and a log rank test were used to assess the survival rate of mice in each group. Other data are expressed as the mean \pm SEM and were analyzed by ANOVA. When results were found to be significant, comparisons were performed using the Bonferroni test. Statistical significance was defined as $P<0.05$.

\section{RESULTS}

Survival rate and $B W$ in experiment 1: According to the Kaplan-Meier analysis, WY14643-treated mice, especially those in the WY14643-early group, had higher mortality than those in the vehicle group in the first 5 days after EMCv inoculation, although the survival rate in the vehicle group decreased rapidly after day 4 and was the first to drop

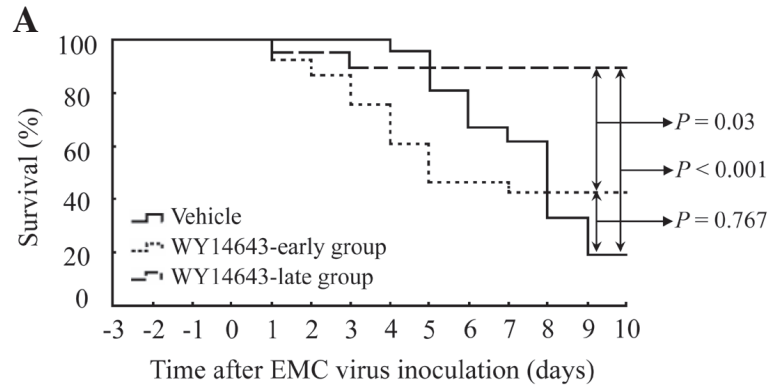

B

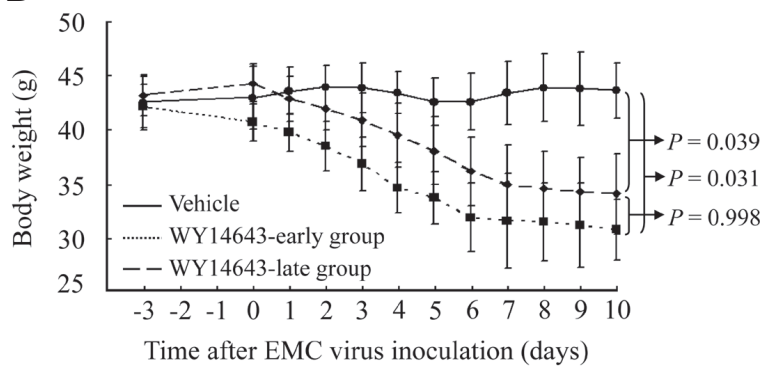

Figure 2. A: Results of the Kaplan-Meier survival analysis showing the lower mortality during the later stages, and the higher mortality during the earlier stages, of myocarditis in the WY14643 treatment groups than in the vehicle group. B: Treatment with WY14643 decreased the body weight of KKAy mice significantly.

Table I. Effects of WY14643 on Body Weight (BW), Heart Weight (HW), Blood Glucose (BG), and Plasma Free Fatty Acid (FFA) in KKAy Mice With Acute Viral Myocarditis

\begin{tabular}{lcccccc}
\hline & $n$ & BW $(\mathrm{g})$ & HW $(\mathrm{mg})$ & HW/BW $(\%)$ & BG $(\mathrm{mmol} / \mathrm{L})$ & FFA $(\mathrm{mEq} / \mathrm{L})$ \\
\hline Day 0 & & & & & & \\
Control & 8 & $38.4 \pm 2.0$ & $152.7 \pm 16.8$ & $0.40 \pm 0.02$ & $7.07 \pm 0.18$ & $1.05 \pm 0.12$ \\
WY14643-early & 8 & $35.4 \pm 2.1^{\#}$ & $132.0 \pm 7.5^{\#}$ & $0.37 \pm 0.03$ & $3.63 \pm 1.05^{\# \#}$ & $1.19 \pm 0.03^{\#}$ \\
Day 4 & & & & & \\
Vehicle & 8 & $38.0 \pm 4.4$ & $174.3 \pm 7.1$ & $0.46 \pm 0.05$ & $14.13 \pm 4.74$ & $1.43 \pm 0.05$ \\
WY14643-early & 8 & $33.1 \pm 2.0^{*}$ & $119.5 \pm 10.1^{* *}$ & $0.36 \pm 0.03^{* *}$ & $1.81 \pm 0.49^{* *}$ & $1.21 \pm 0.09^{*}$ \\
WY14643-late & 8 & $34.8 \pm 0.6^{*}$ & $143.3 \pm 27.5^{* *}$ & $0.41 \pm 0.08$ & $2.28 \pm 1.04^{* *}$ & $1.80 \pm 0.06^{* *}$ \\
Day 9 & & & & & & \\
Vehicle & 7 & $43.6 \pm 2.2$ & $153.4 \pm 13.0$ & $0.35 \pm 0.02$ & $10.66 \pm 2.09$ & $1.84 \pm 0.10$ \\
WY14643-early & 9 & $33.0 \pm 3.3^{* *}$ & $114.3 \pm 9.1^{* *}$ & $0.35 \pm 0.01$ & $6.09 \pm 1.17^{* *}$ & $1.47 \pm 0.13^{* *}$ \\
WY14643-late & 8 & $36.0 \pm 3.6^{*}$ & $141.5 \pm 11.1^{*}$ & $0.40 \pm 0.05$ & $7.32 \pm 2.79^{*}$ & $1.48 \pm 0.16^{* *}$ \\
\hline
\end{tabular}

${ }^{\#} P<0.05,{ }^{\# \#} P<0.01$ with respect to control; ${ }^{*} P<0.05,{ }^{* * *} P<0.01$ with respect to vehicle on the corresponding day; $P<0.01$ with respect to WY14643-early group. 
below 20\% (day 9; Figure 2A). Days 4 and 9 were therefore chosen as the sacrifice days in experiment 2 .

WY14643-early and -late treatment had similar effects on the BW of KKAy mice, with the BW decreasing significantly after the injection of WY14643 to reach statistical significance on day 9 (Figure 2B).

BW and HW in experiment 2: After 3 days of prior treatment, the HW/BW ratio was lower in the WY14643-early group than in the control group, although the difference was not statistically significant. The HW in the two WY14643 treatment groups was markedly lower than in the vehicle group on day 4, although statistical significance was only found between the WY14643-early and vehicle groups after normalization by BW. The HW on day 9 was significantly lower in the two treatment groups than in the vehicle group, whereas HW/BW showed no significant differences between the 3 groups (Table I).

Pathological findings: Myocardial lesions and inflammatory cell infiltration were present in EMCV-inoculated KKAy mice sacrificed on days 4 and 9 (Figure 3 ). The pathological scores (Figure 4A) and number of infiltrating cells (Figure 4B) showed that treatment with WY14643 reduced the se-

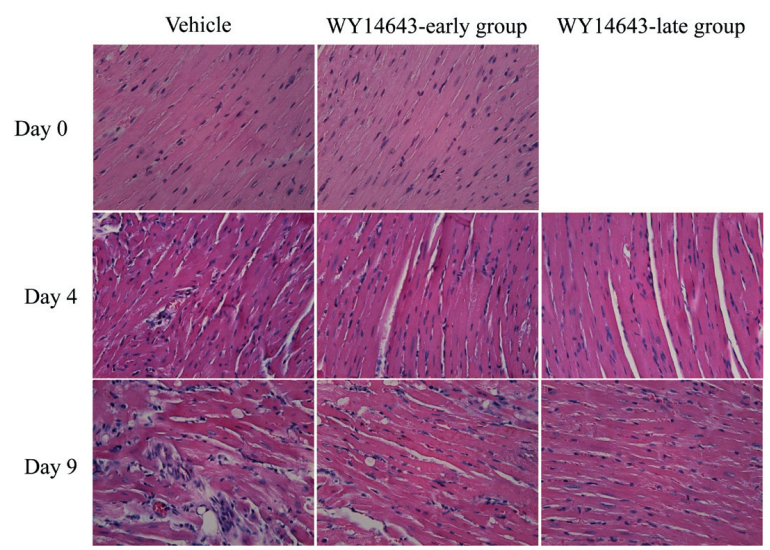

Figure 3. Cardiac pathological findings for KKAy mice on days 0 , 4, and 9. Myocardial necrosis with inflammatory cell infiltration was found after viral inoculation. Treatment with WY14643 reduced the inflammation in heart tissue. verity of the inflammation on both days, and was statistically significant on day 9 .

As shown in Table II, LW, LC, and their ratio (LW/LC) were lower in the WY14643-early group than in control, with the latter two reaching statistical significance. The LW was smaller and the LC larger in the WY14643 treatment groups on day 4 than those in vehicle, with the comparison between the WY14643-late group and vehicle groups reaching statistical significance. The LW/LC ratio was therefore lower in the WY14643 treatment groups than in vehicle on day 4 . The LC in the vehicle group on day 9 was markedly higher than for either the WY14643-early group or -late group, which means that the LW/LC ratio in the vehicle group was significantly smaller than for either of the other two groups. No significant difference was found between the WY14643-early and -late groups as regards LW, LC, and LW/LC on either day 4 or day 9.

The LV myocardial fiber diameters were smaller in the WY14643-early group than in the control on day 0 , although the difference was not statistically significant. The diameters in the WY14643-late group on days 4 and 9 were significantly lower than those in vehicle $(P<0.01)$, and this significance also existed between the vehicle and WY14643-early groups on day 4, although not on day 9. The myocardial diameters in the WY14643-late group were significantly lower than those in the WY14643-early group
A

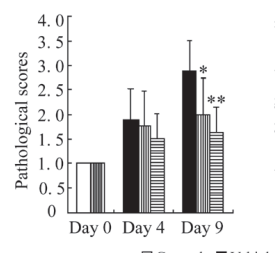

$\square$ Control $\mathbf{a}$
B

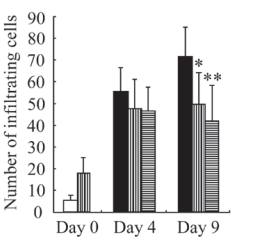

C

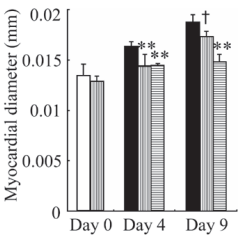

Figure 4. A: The pathological scores for heart tissue; B: the number of infiltration cells at high magnification $(400 \times)$; $\mathbf{C}$ : the diameter of myocardial fibers in KKAy mice. Myocardial necrosis, pathological score, and the number of infiltration cells on day 9 were lower in the two WY14643 treatment groups, especially in the late treatment group. LV myocardial fiber diameters were greater in the vehicle group than in the two WY14643 treatment groups. ${ }^{*} P<0.05,{ }^{* *} P<0.01,{ }^{\dagger} P>0.05$ with respect to vehicle.

Table II. Left Ventricular Wall Thickness (LW) and Cavity Dimension (LC), and Their Ratios

\begin{tabular}{lcccc}
\hline & $n$ & LW $(\mathrm{mm})$ & $\mathrm{LC}(\mathrm{mm})$ & LW/LC \\
\hline Day 0 & & & & \\
Control & 8 & $2.19 \pm 0.45$ & $1.21 \pm 0.53$ & $1.83 \pm 0.36$ \\
WY14643-early group & 8 & $2.01 \pm 0.65$ & $1.51 \pm 0.07^{*}$ & $1.33 \pm 0.49^{\#}$ \\
Day 4 & & & & \\
Vehicle & 8 & $2.23 \pm 0.28$ & $0.57 \pm 0.21$ & $3.84 \pm 2.12$ \\
WY14643-early group & 8 & $1.97 \pm 0.46$ & $0.84 \pm 0.31$ & $2.33 \pm 0.66$ \\
WY14643-late group & 8 & $1.96 \pm 0.44^{*}$ & $0.93 \pm 0.12^{*}$ & $2.11 \pm 0.27^{*}$ \\
Day 9 & & & & \\
Vehicle & 7 & $2.07 \pm 0.22$ & $1.51 \pm 0.29$ & $1.37 \pm 0.25$ \\
WY14643-early group & 9 & $1.95 \pm 0.35$ & $1.35 \pm 0.46^{*}$ & $1.45 \pm 0.63^{*}$ \\
WY14643-late group & 8 & $1.89 \pm 0.53^{*}$ & $1.26 \pm 0.44^{* *}$ & $1.50 \pm 0.55^{* *}$ \\
\hline
\end{tabular}

${ }^{\#} P<0.05$ with respect to control; ${ }^{*} P<0.05,{ }^{* *} P<0.01$ with respect to vehicle on the corresponding day. 
A $\quad \mathrm{TNF} \alpha$

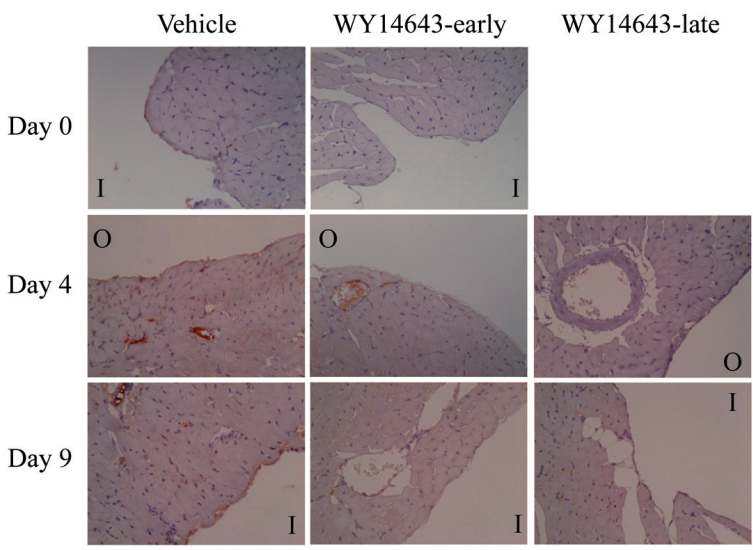

B Adiponectin

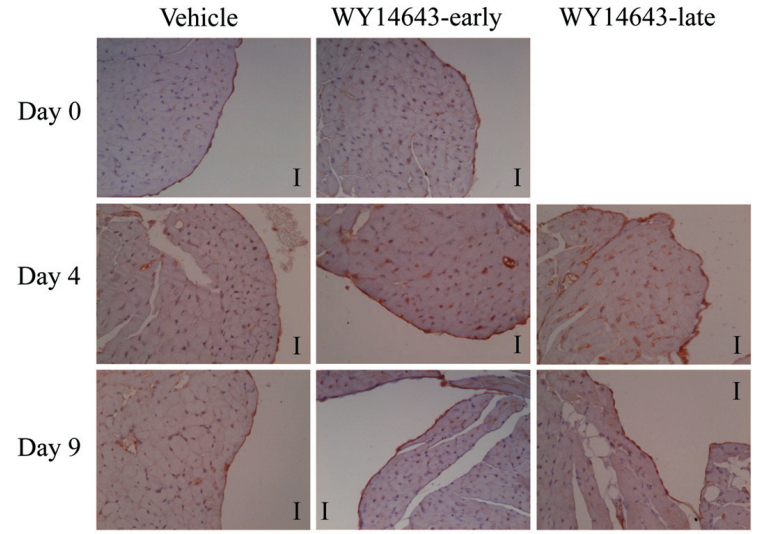

C UCP3

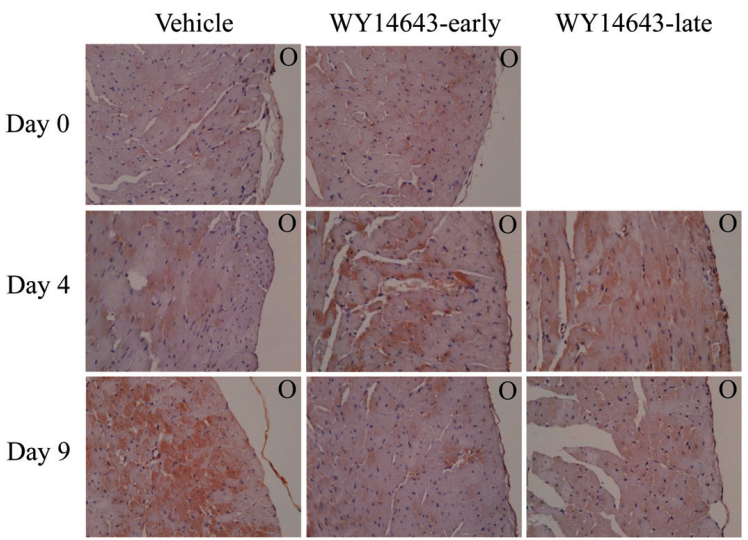

Figure 5. Immunohistochemical findings. Cardiac expression of TNF$\alpha$, adiponectin, and $\mathrm{UCP} 3$ is shown in $\mathrm{A}, \mathrm{B}$, and $\mathrm{C}$ respectively (magnification: $400 \times$ ). I represents inner heart and $\mathrm{O}$ represents outer heart. on day 9 (Figure 4C)

BG and plasma FFA: As shown in Table I, WY14643 treatment significantly ameliorated hyperglycemia in KKAy mice, with no significant difference being found between the two treatment groups. Interestingly, WY14643 increased plasma FFA levels on day 0 in the WY14643-early group and on day 4 in the WY14643-late group, but significantly decreased FFA levels on day 4 and day 9 in the WY14643early group and on day 9 in the WY14643-late group.

Immunohistochemical findings: Cardiac TNF- $\alpha$ was positive in the vehicle group on days 4 and 9 but only weakly positive in the WY14643-early and WY14643-late group (Figure $5 \mathrm{~A})$; cardiac expression of adiponectin protein was more strongly positive in the two WY14643 treatment groups than that in the vehicle group (Figure 5B). Cardiac UCP3 was more strongly positive on day 4 in the two WY14643 treatment groups, but more strongly positive on day 9 in the vehicle group (Figure 5C). Cardiac adiponectin and UCP3 were positive on day 0 , whereas TNF- $\alpha$ was nearly negative in the vehicle and WY14643 treatment groups (Figures 5A, $5 \mathrm{~B}$ and $5 \mathrm{C})$.

TNF- $\alpha$, adiponectin, and UCP3 mRNA expression in heart tissue: The comparative expression levels of TNF- $\alpha$, adiponectin, and UCP3 mRNA in heart tissue on days 0,4 , and 9 after EMCv inoculation are shown in Figure 6. Significantly higher levels of TNF- $\alpha$ mRNA were observed on days 4 and 9 in EMVc-inoculated mice, with WY14643 treatment reducing this increase markedly, especially on day 9. The expression levels of adiponectin mRNA were significantly enhanced by WY14643 treatment on days 0 , 4 , and 9. Three days of prior WY14643 treatment increased the UCP3 mRNA expression levels significantly with re-

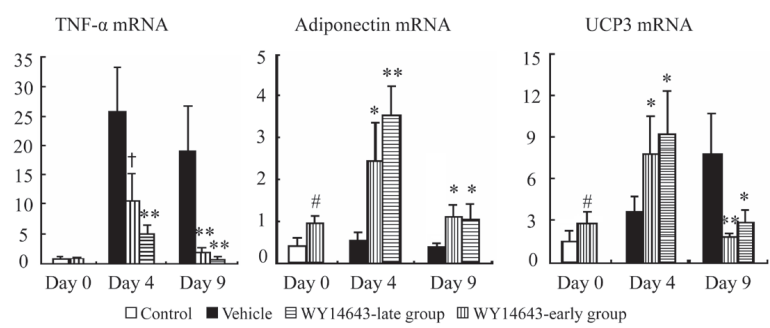

Figure 6. Expression of cardiac TNF- $\alpha$, adiponectin, and UCP3 mRNA by R-PCR. $* P<0.05,{ }^{* *} P<0.01,{ }^{\dagger} P=0.059$ with respect to vehicle; ${ }^{\#} P<0.05$ with respect to control.

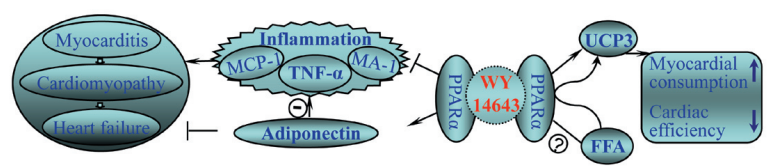

Figure 7. The dual effect of WY14643 on acute viral myocarditis and heart failure in obese diabetic mice. The cardioprotective effect of WY14643 may contribute to reduced inflammatory ${ }^{3)}$ and increased cardiac adiponectin levels, whereas the reduced cardiac efficiency may be due to up-regulation of UCP3 mRNA expression. $\leftarrow$ denotes promotion and - inhibition. MCP-1 indicates monocyte chemoattractant protein-1; MA-1, macrophage antigen-1; FFA, free fatty acid; and $\mathrm{UCP} 3$, uncoupling protein 3 . 
spect to those in control on day 0 . UCP3 mRNA expression levels were significantly higher on day 4 , but lower on day 9 , in the two treatment groups than those in the vehicle group. No significant difference was found between the two WY14643 treatment groups as regards cardiac TNF- $\alpha$, adiponectin, and UCP3 mRNA expression on days 4 and 9.

\section{Discussion}

The above results demonstrate that the $\operatorname{PPAR} \alpha$ agonist WY14643 has different effects on the survival of obese diabetic mice with EMCV-induced heart failure. Thus, simultaneous WY14643 treatment increased the survival rate at the endpoint of experiment 1 mainly due to its anti-inflammatory effects and its enhancement of cardiac adiponectin expression, whereas WY14643-treated mice, especially those in the WY14643-early group, had high mortality in the first 5 days after viral inoculation, possibly as a result of the higher UCP3 levels leading to reduced cardiac efficiency (Figure 7).

The survival rate in our study was similar to that in previous clinical and experimental reports. For example, although fenofibrate, another well-known PPAR $\alpha$ agonist, reduced the risk of nonfatal myocardial infarctions and coronary revascularisations in patients with type 2 diabetes in the FIELD study, sudden cardiac deaths and deaths from heart failure numbered 54 and 11 , respectively, in the placebo group and 70 and 13, respectively, in the fenofibrate group. ${ }^{5)}$ In an animal experiment, Ichihara, et al reported that the survival rate for heart-damaged mice was slightly lower in one of the fenofibrate treatment groups than in the vehicle group at 13 weeks, although the survival rates were significantly higher in the treatment groups than in the vehicle group at 18 weeks. ${ }^{4)}$ All these results suggest that PPAR \pm agonists have a dual effect on the impaired heart.

Inflammation is one of the pivotal factors contributing to the transition from myocarditis and cardiomyopathy to heart failure, therefore, anti-inflammatory treatments play an important role in preventing this transition. ${ }^{17)}$ Recent studies have revealed that WY14643 is capable of inhibiting inflammation in white adipose tissue by suppressing the expression of TNF- $\alpha$, monocyte chemoattractant protein-1, and macrophage antigen-1. ${ }^{3)}$ These inflammatory cytokines are also implicated in myocarditis, dilated cardiomyopathy, and heart failure. ${ }^{18,19)}$ In our recent study, EMCV inoculation into KKAy mice induced severe inflammation in heart tissue and heart failure, which was strongly associated with increased local TNF- $\alpha$ levels. ${ }^{12)}$ In this study, high pathological scores, a large number of infiltrating cells, and a higher $\mathrm{LC} / \mathrm{LW}$ ratio were found in viral-inoculated KKAy mice on day 9. Treatment with WY14643 ameliorated the inflammation in cardiac tissue and reduced the LC/LW ratio by inhibiting TNF- $\alpha$ expression, which might result in a beneficial effect as regards myocarditis, dilated cardiomyopathy, and heart failure, and thereby decrease the mortality.

Adiponectin has recently also been found to be expressed by cardiomyocytes, and the locally produced hormone could be involved in the regulation of cardiac metabolism and function ${ }^{20)}$ and myocardial hypertrophy. ${ }^{21)}$ Our previous study showed that adiponectin was expressed in injured myocytes in autopsy cases ${ }^{22)}$ and that suppressed cardiac adiponectin mRNA expression in obese mice was associated with the development of acute EMCV-induced myocarditis. ${ }^{12)}$ An elevated local expression of adiponectin in cardiac tissue can decrease the severity of myocardial injury associated with the attenuation of cardiac hypertrophy and inflammation in obese mice with acute viral myocarditis. ${ }^{13)}$ In agreement with this previous study, we found that adiponectin mRNA expression levels were markedly higher in WY14643-treated mice on days 0, 4, and 9, and that the myocardial diameter decreased and the inflammation improved. These findings imply that the adiponectin-associated antihypertrophic and anti-inflammatory effects might be involved in WY14643 treatment of EMCv-inoculated KKAy mice.

UCP3 is a recently identified member of the mitochondrial transporter superfamily that is expressed predominantly in heart and skeletal muscle to inhibit the synthesis of ATP in these tissues. ${ }^{23)}$ An elevated expression of local UCP3 has been reported to correlate with increased myocardial oxygen consumption and reduced cardiac efficiency. ${ }^{8)}$ Murray, et al reported that high plasma FFA can increase cardiac UCP3 levels via a PPAR $\alpha$-dependent mechanism. ${ }^{9)}$ Previous reports concerning the effect of WY14643 on plasma FFA levels are contradictory. ${ }^{3,9)}$ For example, Tsuchida, et al reported that an 8-week treatment with WY14643 decreased serum FFA levels significantly in KKAy mice, ${ }^{3)}$ whereas Murray, et al found that a one-week treatment with WY14643 did not reduce plasma FFA levels, although they found a nonsignificant increase in wild-type mice, ${ }^{9)}$ We found that the plasma FFA levels in KKAy mice increased significantly in the WY14643-early group on day 0 (after 3 days of treatment) and in the WY14643-late group on day 4, but decreased significantly in the WY14643-early group after 7 and 12 days of treatment (on days 4 and 9) and in the WY14643-late group after 9 days of treatment (on day 9). The cardiac UCP3 levels therefore appear to vary with plasma FFA levels in a PPAR $\alpha$-dependent manner, as reported by Murray, et al, ${ }^{9)}$ who also found that WY14643 treatment increased myocardial UCP3 levels in wild-type mice by 54\%. In our study, WY14643 treatment increased the expression of UCP3 mRNA in cardiac tissue on days 0 and 4, but decreased it on day 9, which might be due to the combined effect of WY14643 and plasma FFA on PPAR $\alpha$. The increased UCP3 level on day 4 might further decrease ATP synthesis in the inflamed myocardium in WY14643-treated mice, and may therefore be responsible for the lower contractile movement and ATP-dependent iron transport. This would aggravate the heart failure and might be a predictor for the higher mortalities on day 4 in the two WY14643 treatment groups than in vehicle. ${ }^{24}$

As indicated in Table I and Figure 6, 3 days of prior treatment in the WY14643-early group increased plasma FFA levels and cardiac UCP3 mRNA expression, which enhanced myocardial consumption and reduced cardiac ATP production. In the WY14643-late group, however, the plasma FFA levels, cardiac UCP3 mRNA expression, and heart efficiency were the same as those in the control group when attacked by EMCv on day 0, which means that although the heart was protected by the anti-inflammatory effects and increased adiponectin levels equally in the two treatment 
groups during the initial few days of treatment, the heart efficiency was lower in WY14643-early mice. Moreover, WY14643 treatment could significantly replace cardiac n-3 polyunsaturated fatty acids (PUFA) by n-6 PUFA, which would be detrimental to the heart since n-3 PUFAs possess cardioprotective and antiarrhythmic properties. ${ }^{25)}$ In addition, WY14643 treatment might lead to cardiac dysfunction in the diabetic heart by influencing the activity of PPAR $\alpha{ }^{26)}$ These lines of evidence might be the possible foundation for the higher death rate in the WY14643-early group than in the WY14643-late group.

WY14643 treatment also significantly improved the hyperglycemia and reduced the body weight of KKAy mice. It is well known that cardiovascular disease is the leading cause of death for patients with type 2 diabetes and that maintaining glucose homeostasis is crucial for reducing its mortality. ${ }^{27)}$ In addition, an increased body-mass index is also associated with an increased risk of heart failure and death. ${ }^{28)}$ WY14643 therefore appears to improve the survival and heart failure risk for KKAy mice at this point of antihyperglycemia and body weight loss.

The PPAR $\gamma$ signal in mice increased weakly after WY14643 treatment. ${ }^{29)}$ Likewise, PPAR $\gamma$ ligands have been reported to attenuate AngII-induced cardiac fibrosis. ${ }^{30)}$ The pathological findings of this study also showed that treated mice had a lower level of fibrosis. Further studies should therefore aim to determine whether this decrease is due to the slight activation of PPAR $\gamma$ or the activation of PPAR $\alpha$.

PPAR $\alpha$ agonists such as WY14643 and fenofibrate have very complex biological effects resulting from the activation or suppression of dozens of genes, ${ }^{1)}$ the biological effects of which remain largely unknown. Despite the fact that this study only provides limited targets for WY14643, its findings suggest that WY14643 has both beneficial and harmful effects on obese diabetic mice with severe myocarditis and heart failure. In addition, both WY14643 and plasma FFA can increase cardiac UCP3 levels by a PPAR $\alpha$ dependent pathway, and WY14643 can also affect the plasma FFA level. Further studies are therefore required to elucidate the competitive binding of WY14643 and plasma FFA to PPAR $\alpha$ when WY14643 is used as a therapeutic agent.

Conclusion: This study has shown for the first time the cardioprotective and cardio-suppressive effects of WY14643, a potent PPAR \pm agonist, on acute viral myocarditis and heart failure in obese diabetic mice in the initial stages after viral inoculation. The cardioprotective effect of WY14643 may contribute to reduced inflammatory and increased cardiac adiponectin levels, whereas the reduced cardiac efficiency may be due to up-regulation of UCP3 mRNA expression.

\section{ACKNOWLEDGMENTS}

We thank Dr. Hideaki Ninomiya, Department of Pathology, Kanazawa Medical University, for his assistance with the pathological studies, and Ms. Rieko Nozaki and Ms. Miyuki Yoshita, secretaries of the Department of General Medicine, Kanazawa Medical University, for their assistance with ordering reagents and animals.

\section{REFERENCES}

1. Brown JD, Plutzky J. Peroxisome proliferator-activated receptors as transcriptional nodal points and therapeutic targets. Circulation 2007; 115: 518-33. (Review)

2. Takano H, Nagai T, Asakawa M, et al. Peroxisome proliferatoractivated receptor activators inhibit lipopolysaccharide-induced tumor necrosis factor-alpha expression in neonatal rat cardiac myocytes. Circ Res 2000; 87: 596-602.

3. Tsuchida A, Yamauchi T, Takekawa S, et al. Peroxisome proliferator-activated receptor (PPAR) alpha activation increases adiponectin receptors and reduces obesity-related inflammation in adipose tissue: comparison of activation of PPARalpha, PPARgamma, and their combination. Diabetes 2005; 54: 335870.

4. Ichihara S, Obata K, Yamada Y, et al. Attenuation of cardiac dysfunction by a PPAR-alpha agonist is associated with downregulation of redox-regulated transcription factors. J Mol Cell Cardiol 2006; 41: 318-29.

5. Keech A, Simes RJ, Barter P, et al. Effects of long-term fenofibrate therapy on cardiovascular events in 9795 people with type 2 diabetes mellitus (the FIELD study): randomised controlled trial. Lancet 2005; 366: 1849-61.

6. Boudina S, Sena S, Theobald H, et al. Mitochondrial energetics in the heart in obesity-related diabetes: direct evidence for increased uncoupled respiration and activation of uncoupling proteins. Diabetes 2007; 56: 2457-66.

7. Pedraza N, Rosell M, Villarroya J, et al. Developmental and tissue-specific involvement of peroxisome proliferator-activated receptor-alpha in the control of mouse uncoupling protein-3 gene expression. Endocrinology 2006; 147: 4695-704.

8. Boehm EA, Jones BE, Radda GK, Veech RL, Clarke K. Increased uncoupling proteins and decreased efficiency in palmitate-perfused hyperthyroid rat heart. Am J Physiol Heart Circ Physiol 2001; 280: H977-83.

9. Murray AJ, Panagia M, Hauton D, Gibbons GF, Clarke K. Plasma free fatty acids and peroxisome proliferator-activated receptor alpha in the control of myocardial uncoupling protein levels. Diabetes 2005; 54: 3496-502.

10. Bahrami H, Bluemke DA, Kronmal R, et al. Novel metabolic risk factors for incident heart failure and their relationship with obesity: the MESA (Multi-Ethnic Study of Atherosclerosis) study. J Am Coll Cardiol 2008; 51: 1775-83.

11. Matsumori A, Sasayama S. Immunomodulating agents for the management of heart failure with myocarditis and cardiomyopathy--lessons from animal experiments. Eur Heart J 1995; 16: 140-3. (Review)

12. Yu F, Chen R, Takahashi T, et al. Candesartan improves myocardial damage in obese mice with viral myocarditis and induces cardiac adiponectin. Int J Cardiol 2008; 129: 414-21.

13. Takahashi T, Yu F, Saegusa S, et al. Impaired expression of cardiac adiponectin in leptin-deficient mice with viral myocarditis. Int Heart J 2006; 47: 107-23.

14. Kanda T, McManus JE, Nagai R, et al. Modification of viral myocarditis in mice by interleukin-6. Circ Res 1996; 78: 84856.

15. Matsumori A, Kawai C. An experimental model for congestive heart failure after encephalomyocarditis virus myocarditis in mice. Circulation 1982; 65: 1230-5.

16. Kanazawa K, Kawashima S, Mikami S, et al. Endothelial constitutive nitric oxide synthase protein and mRNA increased in rabbit atherosclerotic aorta despite impaired endotheliumdependent vascular relaxation. Am J Pathol 1996; 148: 194956.

17. Mann DL. Inflammatory mediators and the failing heart: past, present, and the foreseeable future. Circ Res 2002; 91: 988-98. (Review)

18. Niu J, Azfer A, Deucher MF, Goldschmidt-Clermont PJ, Kolattukudy PE. Targeted cardiac expression of soluble Fas prevents the development of heart failure in mice with cardiac-specific 
expression of MCP-1. J Mol Cell Cardiol 2006; 40: 810-20.

19. Mann DL. Tumor necrosis factor and viral myocarditis: the fine line between innate and inappropriate immune responses in the heart. Circulation 2001; 103: 626-9.

20. Lopaschuk GD, Folmes CD, Stanley WC. Cardiac energy metabolism in obesity. Circ Res 2007; 101: 335-47. (Review)

21. Shibata R, Ouchi N, Ito M, et al. Adiponectin-mediated modulation of hypertrophic signals in the heart. Nat Med 2004; 10: 1384-9.

22. Takahashi T, Saegusa S, Sumino H, et al. Adiponectin, T-cadherin and tumor necrosis factor-alpha in damaged cardiomyocytes from autopsy specimens. J Int Med Res 2005; 33: 23644.

23. Pedraza N, Rosell M, Villarroya J, et al. Developmental and tissue-specific involvement of peroxisome proliferator-activated receptor-alpha in the control of mouse uncoupling protein-3 gene expression. Endocrinology 2006; 147: 4695-704.

24. Neubauer S, Horn M, Cramer M, et al. Myocardial phosphocreatine-to-ATP ratio is a predictor of mortality in patients with dilated cardiomyopathy. Circulation 1997; 96: 2190-6.
25. Baranowski M, Blachnio-Zabielska A, Gorski J. Peroxisome proliferator-activated receptor alpha activation induces unfavourable changes in fatty acid composition of myocardial phospholipids. J Physiol Pharmacol 2009; 60: 13-20.

26. Finck BN, Han X, Courtois M, et al. A critical role for PPARalpha-mediated lipotoxicity in the pathogenesis of diabetic cardiomyopathy: modulation by dietary fat content. Proc Natl Acad Sci U S A 2003; 100: 1226-31.

27. Panzram G. Mortality and survival in type 2 (non-insulindependent) diabetes mellitus. Diabetologia 1987; 30: 123-31. (Review)

28. Kenchaiah S, Evans JC, Levy D, et al. Obesity and the risk of heart failure. N Engl J Med 2002; 347: 305-13.

29. Buroker NE, Barboza J, Huang JY. The IkappaBalpha gene is a peroxisome proliferator-activated receptor cardiac target gene. FEBS J 2009; 276: 3247-55.

30. Caglayan E, Stauber B, Collins AR, et al. Differential roles of cardiomyocyte and macrophage peroxisome proliferatoractivated receptor gamma in cardiac fibrosis. Diabetes 2008; 57: 2470-9. 